TRANSACTIONS OF THE

AMERICAN MATHEMATICAL SOCIETY

Volume 354, Number 7, Pages 2579-2594

S 0002-9947(02)02908-2

Article electronically published on February 1, 2002

\title{
CORE AND RESIDUAL INTERSECTIONS OF IDEALS
}

\author{
ALBERTO CORSO, CLAUDIA POLINI, AND BERND ULRICH \\ To Professor Craig Huneke on the occasion of his fiftieth birthday
}

\begin{abstract}
D. Rees and J. Sally defined the core of an $R$-ideal $I$ as the intersection of all (minimal) reductions of $I$. However, it is not easy to give an explicit characterization of it in terms of data attached to the ideal. Until recently, the only case in which a closed formula was known is the one of integrally closed ideals in a two-dimensional regular local ring, due to C. Huneke and I. Swanson. The main result of this paper explicitly describes the core of a broad class of ideals with good residual properties in an arbitrary local Cohen-Macaulay ring. We also find sharp bounds on the number of minimal reductions that one needs to intersect to get the core.
\end{abstract}

\section{INTRODUCTION}

Let $R$ be a Noetherian local ring and $I$ one of its ideals. D. Rees and J. Sally defined in 25] the core of $I$, denoted core $(I)$, to be the intersection of all (minimal) reductions of $I$. Roughly, a reduction can be thought of as a simplification of the original ideal: It is a notion introduced by D.G. Northcott and D. Rees [23] and has recently played a crucial role in the study of Rees algebras of ideals. Hence, core $(I)$ can be viewed as the 'simplification of all simplifications' of $I$ ! The core of $I$ naturally appears also in the context of the celebrated Briançon-Skoda theorem, which - in one of its easiest formulations - says that if $R$ is a regular local ring of dimension $d$ and $I$ is an ideal then $\overline{I^{d}} \subset \operatorname{core}(I)$, where $\overline{I^{d}}$ denotes the integral closure of $I^{d}$. Our goal can be framed in terms of giving an explicit description of $\operatorname{core}(I)$ from data attached to the ideal $I$. Without further assumptions, our goal is ambitious - if not hopeless - as core $(I)$ is the intersection of an a priori infinite number of ideals. Thus, our first task is to identify a natural setting where our calculations will go through: Residual intersections of ideals provide such a framework.

To be more specific, an ideal $I$ is said to be integral over an ideal $J \subset I$ if the inclusion of Rees algebras $\mathcal{R}(J) \hookrightarrow \mathcal{R}(I)$ is module finite. The integral closure $\bar{I}$ of $I$ is then defined to be the largest ideal integral over $I$ and the ideal $I$ is integrally closed (or complete) if $\bar{I}=I$. Alternatively, if $I$ is integral over $J$ the ideal $J$ is

Received by the editors April 10, 2001

2000 Mathematics Subject Classification. Primary 13H10; Secondary 13A30, 13B22, 13C40, $13 \mathrm{D} 45$.

Key words and phrases. Integral closure, reductions, residual intersections of ideals.

The first author was partially supported by the NATO/CNR Advanced Fellowships Programme during an earlier stage of this work. The second and third authors were partially supported by the NSF. 
said to be a reduction of $I$. Equivalently, $J$ is a reduction of $I$ if $I^{r+1}=J I^{r}$ for some non-negative integer $r$. The least such $r$ is called the reduction number $r_{J}(I)$ of $I$ with respect to $J$. One then defines the reduction number of $I$ to be the least $r_{J}(I)$, where $J$ varies among all minimal reductions of $I$. A reduction is said to be minimal if it is minimal with respect to containment. Minimal reductions of an ideal have the pleasing property of carrying most of the information about the original ideal but, in general, with fewer generators. If the residue field $k$ of the ring is infinite the minimal number of generators of a minimal reduction equals the analytic spread $\ell$ of the ideal $I$, i.e., the dimension of the special fiber ring $\mathcal{R}(I) \otimes_{R} k$ of $I$.

Little is known about the structure and the properties of core $(I)$. In [25, 2.6] Rees and Sally proved that if $I$ is an $\mathfrak{m}$-primary ideal of a $d$-dimensional local Cohen-Macaulay ring $(R, \mathfrak{m})$ and $\mathcal{A}$ is a 'universal $d$-generated ideal' in $I$, then $\mathcal{A} \cap R \subset \operatorname{core}(I)$. Later, [17, 3.9] provided the first work in the literature with a 'closed formula' for the core of an ideal. More precisely, C. Huneke and I. Swanson showed that the core of integrally closed ideals in two-dimensional regular local rings is still integrally closed and is given by a formula that involves an ideal of minors of any presentation matrix of the ideal. They also showed that the core of such ideals is closely related to the adjoint, introduced by J. Lipman in [21], and proved several arithmetical properties of the core. The techniques used involve a systematic application of the Hilbert-Burch theorem, the notion of quadratic transforms and Rees valuations. More recently, we showed in [9], under fairly general assumptions, for which we refer the reader to [9], that:

- $\operatorname{core}(I)$ is the intersection of finitely many general reductions of $I$;

- $\operatorname{core}(I)$ is the contraction to $R$ of a 'universal ideal in $I$;'

- $\operatorname{core}(I)$ behaves well under flat extensions.

From the second result we also deduced an expression for core $(I)$ as a colon ideal in a polynomial ring over $R$ that allows - at least in principle - for explicit calculations.

The spirit of [9] was close to the one of 25]. In this paper we shift interest instead: Our main goal is to give an explicit formula for core $(I)$ in the spirit of [17], by which we mean a formula that only involves operations inside the ring $R$ itself. The ideals under consideration have slightly more structure than the ones studied in [9]. Nevertheless, our results substantially extend the ones of Huneke and Swanson. The techniques we use are rather different and require some of the machinery developed in [9]. To arrive at our more general formula we needed to observe that residual intersections are the correct objects to replace the Fitting ideals occurring in the Huneke-Swanson result. Thus we can describe the core of ideals $I$ that are balanced (i.e., $J: I$ is independent of the minimal reduction $J$ of $I$, see [27]) or have the expected reduction number $\leq \ell-g+1$, where $g=$ ht $I$. Interestingly enough these conditions also turn out to be necessary for the validity of our formula!

Section 2 contains our main results and their proofs. We let $(R, \mathfrak{m})$ be a local Cohen-Macaulay ring and we focus on ideals that satisfy specific bounds on the number of local generators up to a certain codimension (property $G_{\ell}$ ) and have good residual $S_{2}$ properties. We first prove that for this kind of ideals the property of being balanced is equivalent to the inclusion $(J: I) I \subset \operatorname{core}(I)$, where $J$ is any minimal reduction of $I$ (see Proposition 2.1). We then devote most of the section 
to showing in Theorem 2.6 that this inclusion is indeed an equality, that is

$$
\operatorname{core}(I)=(J: I) I,
$$

for any minimal reduction $J$ of $I$. If in addition $R$ is Gorenstein, $\ell \geq 1$, and $\operatorname{depth} R / I^{j} \geq \operatorname{dim} R / I-j+1$ for $1 \leq j \leq \ell-g+1$, we actually prove that the above formula is equivalent to the ideal $I$ having the expected reduction number. Under these assumptions, we also obtain that core $(I)$ is the intersection of $\ell \cdot\left(\begin{array}{c}\mu(I)-g \\ \ell-g+1\end{array}\right)+1$ general minimal reductions - a bound that is sharp in several cases of interest. To prove Theorem 2.6 we first make a delicate use of residual intersection techniques to reduce to the case of an $\mathfrak{m}$-primary ideal in a one-dimensional local CohenMacaulay ring. We then assemble several intermediate results and lemmas proved earlier as well as a key idea of Huneke and Swanson. We finish the section with an application of Theorem 2.6 which says that the core of a normal balanced ideal is integrally closed (see Theorem 2.11). However, there are examples showing that the integral closedness of $I$ alone is not sufficient to guarantee the one of core $(I)$ (see Example 3.10).

Section 3 lists various classes of ideals for which we can explicitly describe the core from a matrix presenting the ideal. They include the case of perfect ideals of height two or perfect Gorenstein ideals of height three (see Corollaries 3.4, 3.5] 3.6 and 3.7). In particular, Theorem 2.6 recovers [17, 3.9] (see Corollary [3.5]).

In Section 4 we study the core of links of symbolic powers (see Proposition 4.1) and the core of powers of complete intersections (see Proposition 4.2). This result applies to even symbolic powers of self-linked perfect ideals of height 2 and we are able to show that their core is an odd symbolic power of the ideal (see Corollary 4.4). Self-linked ideals have interested many researchers including D. Ferrand, M. Kumar, L. Szpiro, G. Valla and more recently J. Herzog and B. Ulrich [15], and S. Kleiman and B. Ulrich [20]. In particular it was shown in [20] that these ideals are, roughly, in correspondence with Gorenstein perfect algebras of grade one that are birational onto their image - a result that was inspired by recent work in the theory of central projections onto hypersurfaces.

Finally, in Section 5 we discuss Conjecture 5.1: It asks whether - for any nonnilpotent ideal $I$ with $G_{\ell}$ and good residual properties - the core is given by the formula

$$
\operatorname{core}(I)=\left(J^{r}: I^{r}\right) I=\left(J^{r}: I^{r}\right) J=J^{r+1}: I^{r},
$$

for any minimal reduction $J$ of $I$ with reduction number $r$. The point is to move away from the balancedness condition, which was required in Theorem 2.6. This formula has solid theoretical foundation and an extensive computer evidence in its support. Moreover it is sharp, in the sense that [9, 4.11] provides a counterexample if the assumptions are relaxed. We show in Proposition 5.3 that this formula agrees with the one obtained in Theorem [2.6, at least if we add some additional assumptions on the ring $R$ and the powers of the ideal $I$.

\section{Residually $S_{2}$ IDEals}

We begin by reviewing some facts and results from [6] and [9]. Let $R$ be a Noetherian ring, $I$ an $R$-ideal of height $g$, and $s$ an integer. Recall that $I$ satisfies condition $G_{s}$ if for every prime ideal $\mathfrak{p}$ containing $I$ with $\operatorname{dim} R_{\mathfrak{p}} \leq s-1$, the minimal number of generators $\mu\left(I_{\mathfrak{p}}\right)$ of $I_{\mathfrak{p}}$ is at most $\operatorname{dim} R_{\mathfrak{p}}$. The ideal $I$ is said to have property $G_{\infty}$ if $G_{s}$ holds for every $s$. A proper $R$-ideal $K$ is called an $s$-residual 
intersection of $I$, if there exists an $s$-generated ideal $\mathfrak{a} \subset I$ so that $K=\mathfrak{a}: I$ and the height of $K$ is at least $s \geq g$. If in addition the height of $I+K$ is at least $s+1$, then $K$ is said to be a geometric $s$-residual intersection of $I$. We say that $I$ satisfies $A N_{s}\left(A N_{s}^{-}\right)$if $R / K$ is Cohen-Macaulay for every $i$-residual intersection (geometric $i$-residual intersection, respectively) $K$ of $I$ and every $i \leq s$. The ideal $I$ is called $s$-residually $S_{2}$ (weakly s-residually $S_{2}$ ) if $R / K$ satisfies Serre's condition $S_{2}$ for every $i$-residual intersection (geometric $i$-residual intersection, respectively) $K$ of $I$ and every $i \leq s$. Finally, whenever $R$ is local, we say $I$ is universally s-residually $S_{2}$ (universally weakly $s$-residually $S_{2}$ ) if $I S$ is $s$-residually $S_{2}$ (weakly $s$-residually $S_{2}$ ) for every ring $S=R\left(x_{1}, \ldots, x_{n}\right)$ with $x_{1}, \ldots, x_{n}$ variables over $R$.

If $(R, \mathfrak{m})$ is a local Cohen-Macaulay ring of dimension $d$ and $I$ an $R$-ideal satisfying $G_{s}$, then $I$ is universally $s$-residually $S_{2}$ in the following cases:

(1) $R$ is Gorenstein, and the local cohomology modules $H_{\mathfrak{m}}^{d-g-j}\left(R / I^{j}\right)$ vanish for $1 \leq j \leq s-g+1$, or equivalently, $\operatorname{Ext}_{R}^{g+j}\left(R / I^{j}, R\right)=0$ for $1 \leq j \leq s-g+1$ (see [6, 4.1 and 4.3]).

(2) $R$ is Gorenstein, depth $R / I^{j} \geq \operatorname{dim} R / I-j+1$ for $1 \leq j \leq s-g+1$ (see [26. $2.9(\mathrm{a})])$.

(3) $I$ has sliding depth, which means that the $i^{\text {th }}$ Koszul homology modules $H_{i}$ of a generating set $f_{1}, \ldots, f_{n}$ of $I$ satisfy depth $H_{i} \geq \operatorname{dim} R-n+i$ for every $i$ (see [14, 3.3]).

In fact condition (2) implies (1) and the property $A N_{s}$ [26, 2.9(a)]. Assumptions (2) and (3) in turn are satisfied by strongly Cohen-Macaulay ideals, i.e., ideals whose Koszul homology modules are Cohen-Macaulay. This condition always holds if $I$ is a Cohen-Macaulay almost complete intersection or a Cohen-Macaulay deviation two ideal of a Gorenstein ring [2, p. 259]. It is also satisfied for any ideal in the linkage class of a complete intersection [16, 1.11]: Standard examples include perfect ideals of height two and perfect Gorenstein ideals of height three.

The next proposition contains our first result: It relates the shape of the core of $I$ to the property of $I$ being balanced.

Proposition 2.1. Let $R$ be a local Cohen-Macaulay ring with infinite residue field. Let $I$ be an $R$-ideal of height $g$ and analytic spread $\ell$. Suppose that I satisfies property $G_{\ell}$ and is weakly $(\ell-1)$-residually $S_{2}$. Then the following conditions are equivalent:

(a) $(J: I) I \subset \operatorname{core}(I)$ for some minimal reduction $J$ of $I$;

(b) $(J: I) I \subset \operatorname{core}(I)$ for every minimal reduction $J$ of $I$;

(c) $J: I$ does not depend on the minimal reduction $J$ of $I$.

If any of these conditions holds then $I^{2} \subset \operatorname{core}(I)$.

Proof. We may assume that $\ell \neq \mu(I)$. Let $H$ be any minimal reduction of $I$.

If $(a)$ holds then $(J: I) I \subset \operatorname{core}(I) \subset H$, hence $J: I \subset H: I$. Since $I$ is $G_{\ell}$ and weakly $(\ell-1)$-residually $S_{2}, J: I$ and $H: I$ are unmixed ideals of the same dimension $([9,2.1(f)])$. On the other hand $e(R / J: I)=e(R / H: I)$ by $[9,4.2$ and 4.4]. Thus $J: I=H: I$, which gives $(c)$. If $(c)$ holds then $(J: I) I=(H: I) I \subset H$ for every minimal reduction $J$ of $I$, and we obtain (b). Obviously (b) implies $(a)$.

Finally, if any of these conditions holds then by $(c), I \subset H: I$ since $I$ is the sum of its minimal reductions. Hence $I^{2} \subset \operatorname{core}(I)$. 
Our next goal is to strengthen Proposition 2.1 by showing that with essentially the same assumptions one actually has that $\operatorname{core}(I)=(J: I) I$ for any minimal reduction $J$ of $I$. We obtain this result in Theorem 2.6. It requires the assemblage of several facts that we are going to prove next. The proof of Lemma 2.2 below has been inspired by the one of [17, 3.8]. To simplify our notation if $x, y$ are elements of $R$ and $I \subset R$ then by $x: I$ and $x: y$ we mean $(x): I$ and $(x):(y)$, respectively.

Lemma 2.2. Let $(R, \mathfrak{m}, k)$ be a Noetherian local ring. Let $K$ be an $R$-ideal and let $x$ and $y$ be elements of $R$ such that $K y \subset K x$. Assume further that $x$ is a non-zero-divisor. Let $m>\operatorname{dim}_{k}((K: \mathfrak{m}) \cap(x: y) / K)$ and let $u_{1}, \ldots, u_{m}$ be units in $R$ that are not all congruent modulo $\mathfrak{m}$. Then

$$
(K: \mathfrak{m}) x \cap(K: \mathfrak{m})\left(x+u_{1} y\right) \cap \ldots \cap(K: \mathfrak{m})\left(x+u_{m} y\right) \subset(x: y) x .
$$

Proof. Let $\alpha$ be an element of the intersection. Then $\alpha=s x$ with $s \in K: \mathfrak{m}$, but also

$$
\alpha=s_{1}\left(x+u_{1} y\right), \ldots, \alpha=s_{m}\left(x+u_{m} y\right)
$$

with $s_{i} \in K: \mathfrak{m}$. We may assume that $s_{i} \notin K$ for every $i$, because $K y \subset K x$ and $K \subset x: y$. On the other hand, since $\alpha \in(x)$ and $u_{i}$ are units, we have $s_{i} \in x: y$. Using this we wish to show that $s \in x: y$ as well.

Let ' - ' denote images in $\bar{R}=R / K$. Now $\bar{s}_{1}, \ldots, \bar{s}_{m}$, or equivalently, $\bar{u}_{1} \bar{s}_{1}, \ldots$, $\bar{u}_{m} \bar{s}_{m}$ are $m$ nonzero elements of the $k$-vector space $\overline{(K: \mathfrak{m}) \cap(x: y)}$. They are linearly dependent, and shrinking $m$ if needed we may assume that $m$ is minimal with this property. Obviously $m \geq 2$. Now there exist units $\lambda_{1}, \ldots, \lambda_{m}$ in $R$ so that $\sum_{i=1}^{m} \bar{\lambda}_{i} \bar{u}_{i} \bar{s}_{i}=\overline{0}$. Notice that $\sum_{i=1}^{m} \bar{\lambda}_{i} \bar{s}_{i} \neq \overline{0}$ because $u_{1}, \ldots, u_{m}$ are incongruent modulo $\mathfrak{m}$. Since $\sum_{i=1}^{m} \lambda_{i} u_{i} s_{i} \in K$, the element $\sum_{i=1}^{m} \lambda_{i} u_{i} s_{i} y$ is in $K y \subset K x$, and can be written as $\xi x$ for some $\xi \in K$. Set $\lambda=\sum_{i=1}^{m} \lambda_{i}$ and multiply both sides by $\alpha$. Rewriting $\alpha$ by means of the above equations and cancelling $x$ we obtain

$$
\lambda s=\sum_{i=1}^{m} \lambda_{i} s_{i}+\xi \in(x: y)+K=x: y
$$

If $\lambda \in \mathfrak{m}$ then $\lambda s \in K$ since $s \in K: \mathfrak{m}$, and we conclude that $\overline{0}=\sum_{i=1}^{m} \bar{\lambda}_{i} \bar{s}_{i}$, which is impossible. Thus $\lambda$ is a unit, and the desired inclusion $s \in x: y$ follows.

Lemma 2.3. Let $(R, \mathfrak{m})$ be a one-dimensional local Cohen-Macaulay ring with infinite residue field. Let $I$ be an $\mathfrak{m}$-primary ideal. Assume that $J: I$ does not depend on the minimal reduction $J$ of $I$. Then for every minimal reduction $J$ of $I$

$$
\operatorname{core}(I)=(J: I) J .
$$

Proof. By Proposition 2.1 it suffices to show the inclusion core $(I) \subset(J: I) J$. Write $J=(x)$ and $K=x: I$. By assumption, for any other minimal reduction $(y)$ of $I$ one also has $K=y: I$. Moreover, since both $x$ and $y$ are regular elements one can easily check that $(y: I) x=x y: I=(x: I) y$. Thus $K x=K y$. Write $I=\left(y_{1}, \ldots, y_{n}\right)$, 
where $\left(y_{i}\right)$ are minimal reductions of $I$. By Lemma 2.2 there exists $m \gg 0$ and minimal reductions $J_{i j}$ of $I, 1 \leq i \leq n$ and $0 \leq j \leq m$, so that

$$
\bigcap_{j=0}^{m}(K: \mathfrak{m}) J_{i j} \subset\left(x: y_{i}\right) x .
$$

Since $J_{i j}=\left(z_{i j}\right)$ with $z_{i j} R$-regular and $K x=K z_{i j}$, we have $(K x: \mathfrak{m}) \cap J_{i j} \subset$ $(K: \mathfrak{m}) J_{i j}$. Thus

$$
\begin{aligned}
(K x: \mathfrak{m}) \cap \bigcap_{\substack{1 \leq i \leq n \\
0 \leq j \leq m}} J_{i j} & \subset \bigcap_{i=1}^{n} \bigcap_{j=0}^{m}(K: \mathfrak{m}) J_{i j} \subset \bigcap_{i=1}^{n}\left(x: y_{i}\right) x \\
& =\left(\bigcap_{i=1}^{n}\left(x: y_{i}\right)\right) x=(x: I) x=K x .
\end{aligned}
$$

As the ideal $K x$ is $\mathfrak{m}$-primary we deduce that $\bigcap_{\substack{1 \leq i \leq n \\ 0 \leq j \leq m}} J_{i j} \subset K x$, which gives $\operatorname{core}(I) \subset K x=(J: I) J$

Let $R$ be a Noetherian local ring with infinite residue field and let $I$ be an $R$-ideal. We write $\mathcal{M}(I)$ for the set of all minimal reductions of $I$, and define

$$
\gamma(I)=\inf \left\{t \mid \operatorname{core}(I)=\bigcap_{i=1}^{t} J_{i} \text { with } J_{i} \in \mathcal{M}(I)\right\} .
$$

According to [9, 3.1], the number $\gamma(I)$ is finite for a broad class of ideals. In particular it is finite for almost all the ideals considered in the present paper [9] 3.2]. Recall that $I$ is said to be equimultiple if $\ell(I)=$ ht $I$.

Remark 2.4. Let $R$ be a local Cohen-Macaulay ring with infinite residue field and let $I$ be an equimultiple $R$-ideal. If $R_{\mathfrak{p}}$ is Gorenstein for every $\mathfrak{p} \in \operatorname{Min}(I)$, then

$$
\gamma(I)=\max \left\{\operatorname{type}\left((R / \operatorname{core}(I))_{\mathfrak{p}}\right) \mid \mathfrak{p} \in \operatorname{Min}(I)\right\} .
$$

Proof. One has

$$
\begin{aligned}
\gamma(I) & =\max \left(\left\{\gamma\left(I_{\mathfrak{p}}\right) \mid \mathfrak{p} \in \operatorname{Min}\left(\operatorname{Fitt}_{g}(I)\right)\right\} \cup\{1\}\right) \\
& =\max \left\{\gamma\left(I_{\mathfrak{p}}\right) \mid \mathfrak{p} \in \operatorname{Min}(I)\right\}
\end{aligned}
$$

where the first equality follows from $[9,4.9]$ and the second one is a consequence of [10]. On the other hand if $\mathfrak{p} \in \operatorname{Min}(I)$, then $\gamma\left(I_{\mathfrak{p}}\right)=\operatorname{type}\left(R_{\mathfrak{p}} / \operatorname{core}\left(I_{\mathfrak{p}}\right)\right)$ since every minimal reduction of $I_{\mathfrak{p}}$ is an irreducible zero-dimensional $R_{\mathfrak{p}}$-ideal. Finally, $R_{\mathfrak{p}} / \operatorname{core}\left(I_{\mathfrak{p}}\right)=(R / \operatorname{core}(I))_{\mathfrak{p}}$ by $[9,4.8]$.

Lemma 2.5. Let $R$ be a local Cohen-Macaulay ring and let $I$ be an R-ideal. Suppose that I satisfies $G_{s}$ and is weakly $(s-2)$-residually $S_{2}$ for some integer s. Let $K=\mathfrak{a}: I$ be an s-residual intersection of $I$ with $\mathfrak{a} \subset I$ and $\mu(\mathfrak{a}) \leq s$. Then $\mathfrak{a} / K \mathfrak{a}$ is a free $R / K$-module of rank $s$.

Proof. We may assume $s \geq 1$. Let $a_{1}, \ldots, a_{s}$ be a generating sequence of $\mathfrak{a}$ and write

$$
\mathfrak{a}_{i}=\left(a_{1}, \ldots, \widehat{a}_{i}, \ldots, a_{s}\right)
$$


for $1 \leq i \leq s$. By [26, 1.6(a)], $a_{1}, \ldots, a_{s}$ can be chosen so that $\mathfrak{a}_{i}: I$ are $(s-1)$ residual intersections of $I$, and then by [6, 3.1], $\mathfrak{a}_{i}: a_{i}=\mathfrak{a}_{i}: I \subset K$. Hence the coefficients of the relations among $a_{1}, \ldots, a_{s}$ are contained in $K$.

Let $(R, \mathfrak{m}, k)$ be a Noetherian local ring and let $I$ be an $R$-ideal. We say that $J_{1}, \ldots, J_{t}$ are general s-generated ideals in $I$ if $J_{i} \subset I$ are ideals with $\mu\left(J_{i}\right)=s$, $J_{i} \otimes_{R} k \hookrightarrow I \otimes_{R} k$, and the point $\left(J_{1} \otimes_{R} k, \ldots, J_{t} \otimes_{R} k\right)$ lies in some dense open subset of the product of Grassmannians $\stackrel{t}{\times} \mathbb{G}\left(s, I \otimes_{R} k\right)$. For an ideal $I$ of analytic spread $\ell$ we also set $\mathcal{Q}(I)=\left\{\mathfrak{p} \in V\left(\operatorname{Fitt}_{\ell}(I)\right) \mid \operatorname{dim} R_{\mathfrak{p}}=\ell\left(I_{\mathfrak{p}}\right)=\ell\right\}$.

Theorem 2.6. Let $R$ be a local Cohen-Macaulay ring with infinite residue field. Let $I$ be an $R$-ideal of height $g$, analytic spread $\ell$ and minimal number of generators $n$. Suppose that I satisfies $G_{\ell}$ and is universally weakly $(\ell-1)$-residually $S_{2}$. Consider the following conditions:

(a) $(J: I) I \subset \operatorname{core}(I)$ for some minimal reduction $J$ of $I$;

(b) $(J: I) J=(J: I) I=\operatorname{core}(I)$ for every minimal reduction $J$ of $I$;

(c) $J: I$ does not depend on the minimal reduction $J$ of $I$;

(d) the reduction number of $I$ is at most $\ell-g+1$.

They relate in the following way:

(1) Conditions ( $a),(b)$ and $(c)$ are equivalent, and they imply

$$
\gamma(I) \leq \ell \cdot \max \left(\left\{\operatorname{type}\left((R / J: I)_{\mathfrak{p}}\right) \mid \mathfrak{p} \in \mathcal{Q}(I)\right\} \cup\{0\}\right)+1,
$$

where $J$ is any minimal reduction of $I$.

(2) If for every $\mathfrak{p} \in \mathcal{Q}(I), R_{\mathfrak{p}}$ is Gorenstein and $\operatorname{depth}\left(R / I^{j}\right)_{\mathfrak{p}} \geq \ell-g-j+1$ whenever $1 \leq j \leq \ell-g$, then (d) implies the other conditions, and $(a)-(c)$ imply that

$$
\gamma(I) \leq \ell \cdot\left(\begin{array}{c}
n-g \\
\ell-g+1
\end{array}\right)+1
$$

(3) If $\ell \geq 1$ and for every $\mathfrak{p} \in V(I), R_{\mathfrak{p}}$ is Gorenstein and

$$
\operatorname{depth}\left(R / I^{j}\right)_{\mathfrak{p}} \geq \min \left\{\operatorname{dim} R_{\mathfrak{p}}, \ell+1\right\}-g-j+1
$$

whenever $1 \leq j \leq \ell-g+1$, then all four conditions $(a)-(d)$ are equivalent.

Proof. We may assume $\ell \geq 1$. Recall that by [9, 2.1(a) and 4.8], $(\operatorname{core}(I))_{\mathfrak{p}}=$ $\operatorname{core}\left(I_{\mathfrak{p}}\right)$ for every $\mathfrak{p} \in V(I)$. Moreover for any minimal reduction $J$ of $I$ one has ht $J: I \geq \ell$ according to [9, 2.1(f)], and $\operatorname{Ass}_{R}(R / J: I) \subset \mathcal{Q}(I)$ as well as $\operatorname{Ass}_{R}(R / J) \cap V(J: I) \subset \mathcal{Q}(I)$ by [6, 3.4(a),(b)] and [9, 2.1(e),(g)].

We now prove (1). In light of Proposition 2.1, to establish the asserted equivalence it is enough to show that $(c)$ implies core $(I) \subset(J: I) J$ for $J$ any minimal reduction of $I$. It suffices to prove the inclusion $\operatorname{core}(I) \subset(J: I) J$ locally at every associated prime $\mathfrak{p}$ of the latter ideal. We may assume that $\mathfrak{p}$ contains $J: I$, since the desired inclusion is clear otherwise. Now according to Lemma 2.5 $\operatorname{Ass}_{R}(R /(J: I) J) \subset \operatorname{Ass}_{R}(R /(J: I)) \cup \operatorname{Ass}_{R}(R / J)$. Hence by the above we may assume that $\mathfrak{p} \in \mathcal{Q}(I)$. Furthermore [9. 2.1(e) and 4.9] shows that $\gamma(I) \leq \max \left(\left\{\gamma\left(I_{\mathfrak{p}}\right) \mid \mathfrak{p} \in \mathcal{Q}(I)\right\} \cup\{1\}\right)$. Finally by [9, 2.1(a)] and Proposition 2.1. our assumptions are preserved as we replace $R$ by $R_{\mathfrak{p}}$. Thus we may from now on assume that $R=R_{\mathfrak{p}}$ with $\mathfrak{p}$ in $\mathcal{Q}(I)$.

Let $a_{1}, \ldots, a_{\ell}$ be a generating sequence of $J$ and write $\mathfrak{a}_{i}=\left(a_{1}, \ldots, \widehat{a}_{i}, \ldots, a_{\ell}\right)$, $K_{i}=\mathfrak{a}_{i}: I$ for $1 \leq i \leq \ell$. By [26, 1.6(a)], $a_{1}, \ldots, a_{\ell}$ can be chosen so that $K_{i}$ are geometric $(\ell-1)$-residual intersections. 
For a fixed $i$ let ' - ' denote images modulo $K_{i}$. According to [6, 3.4, 3.1 and $2.4(b)], \bar{R}$ is a one-dimensional local Cohen-Macaulay $\operatorname{ring}, \bar{I}$ is a height one ideal, $K_{i} \cap I=\mathfrak{a}_{i}$ and $\bar{J}: \bar{R} \bar{I}=\overline{J:_{R} I}$. We now claim that $\overline{\operatorname{core}(I)} \subset \operatorname{core}(\bar{I})$. By [9] $4.5]$, for $t=\gamma(\bar{I})$ general one-generated ideals $\left(\alpha_{1}\right), \ldots,\left(\alpha_{t}\right)$ in $I$ one has core $(\bar{I})=$ $\left(\bar{\alpha}_{1}\right) \cap \ldots \cap\left(\bar{\alpha}_{t}\right)$. As $\left(\mathfrak{a}_{i}, \alpha_{j}\right)$ are reductions of $I$, we obtain $\operatorname{core}(I) \subset \bigcap_{j=1}^{t}\left(\mathfrak{a}_{i}, \alpha_{j}\right)$. Therefore

$$
\overline{\operatorname{core}(I)} \subset \overline{\bigcap_{j=1}^{t}\left(\mathfrak{a}_{i}, \alpha_{j}\right)} \subset \bigcap_{j=1}^{t}\left(\bar{\alpha}_{j}\right)=\operatorname{core}(\bar{I})
$$

By Proposition 2.1 $(J: I) I \subset \operatorname{core}(I)$. Therefore $(\bar{J}: \bar{R} \bar{I}) \bar{I}=\overline{\left(J:_{R} I\right) I} \subset \overline{\operatorname{core}(I)} \subset$ core $(\bar{I})$. Thus, again using Proposition 2.1 we deduce that $H: \bar{I}$ does not depend on the minimal reduction $H$ of $\bar{I}$. Now Lemma 2.3 implies that $\operatorname{core}(\bar{I})=(\bar{J}: \bar{I}) \bar{J}$. Thus $\overline{\operatorname{core}(I)} \subset \overline{(J: I) J}$, which yields core $(I) \subset(J: I) J+K_{i}$.

It follows that

$$
\operatorname{core}(I) \subset \bigcap_{1 \leq i \leq \ell}\left(\left[(J: I) J+K_{i}\right] \cap I\right)=\bigcap_{1 \leq i \leq \ell}\left((J: I) J+\mathfrak{a}_{i}\right) .
$$

Now, using Lemma 2.5 we conclude that $\bigcap_{1 \leq i \leq \ell}\left((J: I) J+\mathfrak{a}_{i}\right)=(J: I) J$. Therefore core $(I) \subset(J: I) J$, which proves the asserted equivalence.

To establish the bound for $t=\gamma(I)$, write core $(I)=J_{1} \cap \ldots \cap J_{t}$ where $J_{1}, \ldots, J_{t}$ are minimal reductions of $I$. By $(b), \operatorname{core}(I)=\left(J_{1}: I\right) J_{1}$. Since the module $M=$ $J_{1} / \operatorname{core}(I)=J_{1} /\left(J_{1}: I\right) J_{1}$ is Artinian, $t-1 \leq \operatorname{type}(M)$. On the other hand Lemma 2.5 shows that type $(M)=\ell \cdot \operatorname{type}\left(R / J_{1}: I\right)$. Finally by $(c), J_{1}: I=J: I$, completing the proof of the inequality $\gamma(I) \leq \ell \cdot \operatorname{type}(R / J: I)+1$.

We now prove $(2)$. In showing that $(d)$ implies $(a)$ it suffices to verify $(a)$ locally at every associated prime $\mathfrak{p}$ of $\operatorname{core}(I)$. Since by $[9,3.1,3.2] \operatorname{core}(I)$ is a finite intersection of minimal reductions of $I$, we may assume that $\mathfrak{p} \in \mathcal{Q}(I)$. If $\ell=g$ then obviously ht $I_{\mathfrak{p}}=g$, whereas for $\ell>g, \ell-\operatorname{ht} I_{\mathfrak{p}}=\operatorname{dim}(R / I)_{\mathfrak{p}} \geq \operatorname{depth}(R / I)_{\mathfrak{p}} \geq \ell-g$. Thus in either case ht $I_{\mathfrak{p}}=g$. Also notice that the reduction number of $I$ cannot increase upon localizing at $\mathfrak{p}$ since $\mathfrak{p} \in \mathcal{Q}(I)$. Now, replacing $R$ by $R_{\mathfrak{p}}$ we may suppose that $R$ is Gorenstein of dimension $\ell$ and depth $R / I^{j} \geq \ell-g-j+1$ for $1 \leq$ $j \leq \ell-g$, hence for $1 \leq j \leq \ell-g+1$. In this situation, [27, 2.6] shows that $(d)$ implies $(c)$, which in turn yields $(a)$ by Proposition 2.1. Finally, the asserted inequality for $\gamma(I)$ follows from $(1)$ and the fact that type $\left((R / J: I)_{\mathfrak{p}}\right) \leq\left(\begin{array}{c}n-g \\ \ell-g+1\end{array}\right)$ for $\mathfrak{p} \in \mathcal{Q}(I)$ by $[26,2.9(b)]$.

To prove $(3)$ we need to show that $(c)$ implies $(d)$. But this follows from [24, 3.3] (see also [27, 4.8]).

Remark 2.7. In the setting of Theorem 2.6 core $(I)$ is the intersection of $\gamma(I)$ general $\ell$-generated ideals in $I$ which are reductions of $I$ (see [9, 4.5]).

Remark 2.8. Theorem 2.6 is essentially a cancellation theorem (see 8 for details). Indeed, the fact that $(b)$ implies $(c)$ as asserted in Theorem 2.6 can also be restated by saying that $\left(J_{1}: I\right) I=\left(J_{2}: I\right) I$ implies $J_{1}: I=J_{2}: I$ for any minimal reductions $J_{1}$ and $J_{2}$ of $I$. 
Remark 2.9. Given two ideals $I$ and $J$ of $R$, the coefficient ideal $\mathfrak{a}(I, J)$ of $I$ with respect to $J$ is the largest ideal $\mathfrak{a}$ of $R$ such that $\mathfrak{a} J=\mathfrak{a} I$. This ideal has been introduced by I.M. Aberbach and C. Huneke [1, 2.1] and has played an important role in theorems of Briançon-Skoda type with coefficients [1, 2.7]. Clearly, $\mathfrak{a}(I, J) \subset J: I$. If, in addition, $I$ satisfies one of the equivalent conditions $(a)-(c)$ in Theorem 2.6 then $\mathfrak{a}(I, J)=J: I$ for any minimal reduction $J$ of $I$; in particular $\mathfrak{a}(I, J)$ does not depend on the minimal reduction $J$ of $I$. This was already known in the case $\ell=g[7,2.1]$.

Remark 2.10. Closely related is also the notion of the adjoint of $I$, denoted adj $(I)$, introduced by J. Lipman [21]. If $R$ is a regular local ring essentially of finite type over a field of characteristic zero and $I$ is an $R$-ideal with analytic spread $\ell$, Lipman shows that $\operatorname{adj}\left(I^{\ell-1}\right) \subset \mathfrak{a}(I, J)$ for any minimal reduction $J$ of $I$ [21, 2.3]. Furthermore if $(R, \mathfrak{m})$ is a regular local ring of dimension two with infinite residue field and $I$ is an integrally closed $\mathfrak{m}$-primary ideal, he proves that this containment is an equality [21,3.3], and Huneke-Swanson deduce that $\operatorname{core}(I)=\operatorname{adj}(I) I$ [17, 3.14]. Combining [18, 3.5], Remark 2.9 and Theorem 2.6(2) one obtains the following more general result: Let $R$ be a regular local ring essentially of finite type over a field of characteristic zero. Let $I$ be an $R$-ideal of height $g$ and analytic spread $\ell$. Suppose that $I$ satisfies $G_{\ell}$, the reduction number of $I$ is at most $\ell-g+1$, $\operatorname{depth} R / I^{j} \geq \operatorname{dim} R / I-j+1$ whenever $1 \leq j \leq \ell-g+1$, and $\operatorname{Proj}(\mathcal{R}(I))$ has rational singularities. Then $\operatorname{core}(I)=\operatorname{adj}\left(I^{g-1}\right) I$.

Huneke and Swanson were in part motivated to study the core because the core of an integrally closed ideal in a two-dimensional regular local ring is still integrally closed (see [17 3.12]). The following theorem fully generalizes the one of Huneke and Swanson, as it is a celebrated result of O. Zariski that integrally closed ideals in a two-dimensional regular local ring are normal [29, p. 385]. We recall that $I$ is said to be normal if $\overline{I^{j}}=I^{j}$ for all $j \geq 0$.

Theorem 2.11. Let $R$ be a local Cohen-Macaulay ring with infinite residue field and let $I$ be an $R$-ideal with analytic spread $\ell$. Suppose that ht $I>0, I$ satisfies $G_{\ell}$ and $A N_{\ell-1}^{-}$, and $I$ is universally weakly $(\ell-1)$-residually $S_{2}$. Further assume that one of the equivalent conditions $(a)-(c)$ of Theorem 2.6 holds. Let $J$ be a minimal reduction of $I$.

(1) The $\mathcal{R}(I)$-module $(J: I) \mathcal{R}(I)$ satisfies $S_{2}$. This module is a maximal CohenMacaulay $\mathcal{R}(I)$-module if I satisfies $A N_{\ell}$.

(2) Assume in addition that $R$ is a normal ring and $I$ is a normal ideal. Then the ideal $(J: I) I^{j}$ is integrally closed for every $j \geq 0$. In particular $J: I$ and core $(I)$ are integrally closed.

Proof. Write $d=\operatorname{dim} R$ and $K=J: I$.

To prove (1) it suffices to show that as an $\mathcal{R}(J)$-module, $K \mathcal{R}(I)$ satisfies $S_{2}$ or is maximal Cohen-Macaulay, respectively. Condition $(b)$ of Theorem 2.6 implies that $K \mathcal{R}(I)=K \mathcal{R}(J)$. According to [9] 2.1(f)], ht $K \geq \ell$. Therefore $J$ satisfies $G_{\infty}$ and $A N_{\ell-1}^{-}$by [26, 1.12], and then $J$ has the 'sliding depth property' by [26, 1.8(c)]. Now [13, 9.1] shows that $\mathcal{R}(J) \simeq \operatorname{Sym}_{R}(J)$ is a Cohen-Macaulay ring, necessarily of dimension $d+1$. Thus we may assume that $K \neq R$. Now $K$ is unmixed of height $\ell$ according to [26, 1.7(a)], and if $I$ satisfies $A N_{\ell}$ then $R / K$ is Cohen-Macaulay. On the other hand Lemma 2.5 gives that $J / K J$ is a free $R / K$-module of rank $\ell$, and therefore $\mathcal{R}(J) / K \mathcal{R}(J) \simeq \operatorname{Sym}_{R / K}(J / K J)$ is a polynomial ring over $R / K$ in 
$\ell$ variables. Thus $\mathcal{R}(J) / K \mathcal{R}(J)$ is $S_{1}$ and equidimensional of dimension $d$, and if $I$ satisfies $A N_{\ell}$ then this ring is Cohen-Macaulay. As $\mathcal{R}(J)$ is Cohen-Macaulay of dimension $d+1$ we deduce that the $\mathcal{R}(J)$-module $K \mathcal{R}(J)$ satisfies $S_{2}$ or is maximal Cohen-Macaulay, respectively.

We now prove (2). By part (1), $K \mathcal{R}(I)$ is a divisorial ideal of the normal domain $\mathcal{R}(I)$. Such an ideal is necessarily integrally closed. Since $K \mathcal{R}(I)=\bigoplus_{j \geq 0} K I^{j} t^{j}$ we conclude that the $R$-ideals $K I^{j}$ are integrally closed as well (for a similar argument see [12] the proof of 1.5$]$ ).

\section{EXAMPLES}

In this section, we illustrate Theorems 2.6 and 2.11 with a sequence of examples.

Example 3.1. Good ideals in the sense of S. Goto, S.-I. Iai and K.-I. Watanabe [1] - by which we mean $\mathfrak{m}$-primary ideals $I$ of a local Gorenstein ring $(R, \mathfrak{m})$ such that $I=J: I$ and $I^{2}=J I$ for some complete intersection ideal $J$-are examples of ideals for which core $(I)=I^{2}$.

Example 3.2. Let $R$ be a two-dimensional regular local ring with infinite residue field. It is well known that all powers of the maximal ideal $\mathfrak{m}=(x, y)$ have reduction number at most one. From Theorem [2.6(2) we obtain

$$
\operatorname{core}\left(\mathfrak{m}^{j}\right)=\left(\left(x^{j}, y^{j}\right): \mathfrak{m}^{j}\right) \mathfrak{m}^{j}=\mathfrak{m}^{j-1} \mathfrak{m}^{j}=\mathfrak{m}^{2 j-1} .
$$

In particular, $\gamma\left(\mathfrak{m}^{j}\right)=2 j-1$ by Remark 2.4. This shows that the bound in Theorem 2.6 2) is sharp and that there is no upper bound for $\gamma(I)$ as $I$ varies among the ideals of $R$. If the restriction on the dimension is dropped, one can still compute the core of $\mathfrak{m}^{j}$, although Theorem 2.6 may no longer apply, see Proposition 4.2 .

Example 3.3. Let $R=k[x, y, z]_{(x, y, z)}$ with $k$ a field of characteristic zero and $x, y, z$ variables, and let $\mathfrak{m}$ denote the maximal ideal of $R$. Consider the Gorenstein $R$-ideal of height three

$$
I=\left(x^{2}-y^{2}+x z, x y+x z-y z, x z-2 y z+z^{2}, y^{2}+y z-z^{2}, z^{2}-2 y z\right),
$$

and let $J$ be the $R$-ideal generated by the first three generators of $I$. The ideal $I$ has reduction number 2 . Hence it fails to satisfy conditions $(a)-(d)$ of Theorem 2.6 However, by [9, 4.5] the core of $I$ is the intersection of homogeneous minimal reductions of $I$. So it will definitely contain $\mathfrak{m}^{4}$. On the other hand, taking 10 general homogeneous minimal reductions $J_{1}, \ldots, J_{10}$ of $I$ a calculation using the computer algebra system Macaulay shows that $J_{1} \cap \ldots \cap J_{10}=\mathfrak{m}^{4}$. Therefore $\operatorname{core}(I)=\mathfrak{m}^{4}$.

In the case of perfect ideals of height two or perfect Gorenstein ideals of height three one can compute the core explicitly from a matrix presenting $I$.

Corollary 3.4. Let $R$ be a local Gorenstein ring with infinite residue field. Let I be a perfect $R$-ideal of height two and analytic spread $\ell$, satisfying $G_{\ell}$. Let $\varphi$ be a matrix with $n$ rows presenting $I$. Then the following conditions are equivalent:

(a) the reduction number of $I$ is at most $\ell-1$;

(b) $\operatorname{core}(I)=I_{n-\ell}(\varphi) \cdot I$.

If any of these conditions holds and if $R$ and $I$ are normal, then $I_{n-\ell}(\varphi)$ and $\operatorname{core}(I)$ are integrally closed.

Proof. The result follows from [5] and Theorems [2.6(3) and 2.11]2). 
Corollary 3.5 (see [17, 3.9]). Let $R$ be a two-dimensional regular local ring with infinite residue field. Let $I$ be an integrally closed $R$-ideal and let $\varphi$ be a matrix with $n$ rows presenting $I$. Then

$$
\operatorname{core}(I)=I_{n-2}(\varphi) \cdot I \text {. }
$$

Furthermore $I_{n-2}(\varphi)$ and core $(I)$ are integrally closed.

Proof. We may assume that $I$ is primary to the maximal ideal of $R$. The ideal $I$ has reduction number at most one by [22, 5.5] and is normal according to [29, p. 385]. Now the assertion follows from Corollary [3.4.

Corollary 3.6. Let $R$ be a local Gorenstein ring with infinite residue field. Let $I$ be a perfect Gorenstein $R$-ideal of height three, analytic spread $\ell$ and minimal number of generators $n$, satisfying $G_{\ell}$. Let $\varphi$ be a matrix with $n$ rows presenting $I$. Then the following conditions are equivalent:

(a) the reduction number of $I$ is $\ell-2$;

(b) $\operatorname{core}(I)=I_{1}(\varphi) \cdot I$.

If any of these conditions holds and if $R$ and $I$ are normal, then $I_{1}(\varphi)$ and core $(I)$ are integrally closed.

Proof. The result follows from [27, 5.6], [19, 5.5] and Theorems 2.6.(3) and 2.11(2).

Corollary 3.7. Let $R$ be a local Gorenstein ring with infinite residue field. Let I be an $R$-ideal of height $g$, analytic spread $\ell$, minimal number of generators $n=\ell+1 \geq$ 2 , satisfying $G_{\ell}$ and $\operatorname{depth} R / I^{j} \geq \operatorname{dim} R / I-j+1$ for $1 \leq j \leq \ell-g+1$. Let $\varphi$ be a matrix with $n$ rows presenting $I$. Then the following conditions are equivalent:

(a) the reduction number of $I$ is $\ell-g+1$;

(b) $\operatorname{core}(I)=I_{1}(\varphi) \cdot I$.

If any of these conditions holds and if $R$ and $I$ are normal, then $I_{1}(\varphi)$ and core $(I)$ are integrally closed.

Proof. The result follows from [19, 5.5] and Theorems 2.6(3) and 2.11(2).

Remark 3.8. In the setting of Corollaries 3.4, 3.6] and 3.7, [27, 2.6] (or Theorem [2.6(2)) provides additional simplification in the computation of the core of $I$. In the case of Corollary $3.4(b)$ we have that after elementary row operations, $I_{n-\ell}(\varphi)$ is generated by the $n-\ell$ by $n-\ell$ minors of the matrix consisting of the last $n-\ell$ rows of $\varphi$. In the case of Corollaries $3.6(b)$ and 3.7 $(b)$ we have that after elementary row operations, $I_{1}(\varphi)$ is generated by the entries of the last row of $\varphi$.

The next example is an illustration of Corollary 3.7 In this situation the integral closedness of the ideal alone suffices to prove the one of the core.

Example 3.9. Let $R=k\left[x_{1}, \ldots, x_{d}\right]_{\left(x_{1}, \ldots, x_{d}\right)}$ with $k$ an infinite field and $x_{1}, \ldots$, $x_{d}$ variables, and let $\mathfrak{m}$ denote the maximal ideal of $R$. Let $I$ be an $R$-ideal of height $g$ and minimal number of generators $d+1 \geq 2$, satisfying $G_{d}$ and $\operatorname{depth} R / I^{j} \geq \operatorname{dim} R / I-j+1$ for $1 \leq j \leq d-g$. Assume that $I$ has a presentation matrix whose entries are linear forms in $k\left[x_{1}, \ldots, x_{d}\right]$. Then $\operatorname{core}(I)=\mathfrak{m} I$. If $I$ is integrally closed then so is core $(I)$. 
Proof. Let $\varphi$ be a matrix of linear forms presenting $I$. Notice that after elementary row operations, the entries of the last row of $\varphi$ generate $\mathfrak{m}$. Now by [27, 5.1], $\ell(I)=d$ and condition $(a)$ of Corollary 3.7 holds. Since $I_{1}(\varphi)=\mathfrak{m}$, the corollary shows that $\operatorname{core}(I)=\mathfrak{m} I$. The ideal $I$ is generated by forms in $k\left[x_{1}, \ldots, x_{d}\right]$ of the same degree, say $s$. Therefore $\mathfrak{m} I=\mathfrak{m}^{s+1} \cap I$, and it follows that $\operatorname{core}(I)$ is integrally closed if $I$ is.

Given the previous example, one may hope that a more general analogue of Theorem 2.11 2) - where the normality of the ideal $I$ is replaced by $I$ being integrally closed - holds. The following example however shows that this is not true in general.

Example 3.10. Let $R=k[x, y, z, w]_{(x, y, z, w)}$ with $k$ a field of characteristic zero. Let $I$ be the $R$-ideal generated by the four by four Pfaffians of the five by five alternating matrix

$$
\varphi=\left(\begin{array}{ccccc}
0 & -x^{2} & -y^{2} & -z^{2} & -w^{2} \\
x^{2} & 0 & -w^{2} & y^{2} & -z^{2} \\
y^{2} & w^{2} & 0 & -x^{2} & -x^{2} \\
z^{2} & -y^{2} & x^{2} & 0 & -y^{2} \\
w^{2} & z^{2} & x^{2} & y^{2} & 0
\end{array}\right)
$$

The ideal $I$ is perfect Gorenstein of height three. Since $I$ is generically a complete intersection and $I_{1}(\varphi)$ is generated by the entries of the last row of $\varphi$, by [27, 5.1] $I$ satisfies condition (a) of Corollary 3.6. Thus one has that core $(I)=\left(x^{2}, y^{2}, z^{2}, w^{2}\right)$. $I$. A computation using the computer algebra system Macaulay shows that $I=\sqrt{ } I$. Hence the ideal $I$ is integrally closed. However, core $(I)$ is not an integrally closed ideal: For example, the element $x w\left(x^{4}+y^{4}+z^{2} w^{2}\right) \notin \operatorname{core}(I)$, but it is integral over core $(I)$.

\section{Symbolic POWERS OF IDEALS}

Let $I$ be an ideal in a Noetherian ring $R$, let $W$ be the complement in $R$ of the union of all the associated primes of $I$, and let $j \geq 1$ be an integer. We recall that the $j^{\text {th }}$ symbolic power $I^{(j)}$ of $I$ is the preimage of $I^{j} R_{W}$ in $R$. In this section we study the core of links of symbolic powers of ideals. We also compute the core of powers of complete intersections. Finally, we apply the latter result to the case of even symbolic powers of self-linked ideals.

Proposition 4.1. Let $R$ be a local Gorenstein ring with infinite residue field. Let $H$ be an unmixed $R$-ideal of height $g \geq 2$ which is generically a complete intersection. Let $\underline{\alpha}=\alpha_{1}, \ldots, \alpha_{g}$ be a regular sequence contained in $H^{(j)}$ and set $I=(\underline{\alpha}): H^{(j)}=$ $(\underline{\alpha}): H^{j}$ for some $j \geq 1$. If $g=2$ or $j=1$, assume that at least two of the $\alpha_{i}$ 's are contained in $H^{(j+1)}$. Then

$$
\operatorname{core}(I)=H^{(j)} I \text {. }
$$

Proof. From [24, 4.1] we have $I^{2}=(\underline{\alpha}) I$. Hence Theorem [2.6 (2) shows that $\operatorname{core}(I)=((\underline{\alpha}): I) I=H^{(j)} I$.

Sometimes the core of (symbolic) powers can be computed directly even if the reduction number is not the expected one: 
Proposition 4.2. Let $R$ be a Noetherian local ring with infinite residue field. Let $I$ be an $R$-ideal of height $g \geq 1$ generated by a regular sequence. Then for any $j \geq 1$,

$$
\begin{array}{r}
\operatorname{core}\left(I^{j}\right)=I^{g j-g+1} . \\
\text { If } R \text { is Gorenstein one has } \gamma\left(I^{j}\right)=\left(\begin{array}{c}
g j-1 \\
g-1
\end{array}\right) .
\end{array}
$$

Proof. The second assertion is an immediate consequence of Remark 2.4. To prove the first one write $\mathfrak{m}$ for the maximal ideal of $R, k=R / \mathfrak{m}, A=R / I, \mathfrak{n}=\mathfrak{m} / I, \mathcal{G}=$ $\operatorname{gr}_{I}(R)$, and $\mathcal{F}=\operatorname{gr}_{I}(R) \otimes_{R} k$. Notice that $\mathcal{G} \simeq A\left[x_{1}, \ldots, x_{g}\right]$ and $\mathcal{F} \simeq k\left[x_{1}, \ldots, x_{g}\right]$ are polynomial rings in $g$ variables with irrelevant ideals $\mathcal{G}_{+}=\left(x_{1}, \ldots, x_{g}\right) \mathcal{G}$ and $\mathcal{F}_{+}=\left(x_{1}, \ldots, x_{g}\right) \mathcal{F}$, respectively.

Let $J$ be any minimal reduction of $I^{j}$ and set $J^{\prime}=J+\mathfrak{m} I^{j} / \mathfrak{m} I^{j} \subset[\mathcal{F}]_{j}$. The equality $\left(I^{j}\right)^{r+1}=J\left(I^{j}\right)^{r}$ for $r \gg 0$ implies that $\sqrt{J^{\prime} \mathcal{F}}=\mathcal{F}_{+}$. Thus $J^{\prime} \mathcal{F}$ is a complete intersection $\mathcal{F}$-ideal minimally generated by $g$ forms of degree $j$. Computing the socle degree of $\mathcal{F} / J^{\prime} \mathcal{F}$, we see that $\left(\mathcal{F}_{+}\right)^{g j-g+1} \subset J^{\prime} \mathcal{F}$. Thus $I^{g j-g+1}=$ $J I^{g j-g-j+1}$ by Nakayama's Lemma. This proves the inclusion $I^{g j-g+1} \subset \operatorname{core}\left(I^{j}\right)$.

To show the opposite containment, let $u \in R \backslash I^{g j-g+1}$. We need to find a minimal reduction of $I^{j}$ that does not contain $u$. For this we may suppose $g j-g \geq j$. Furthermore, as $\mathcal{G}$ is a polynomial ring we may replace $u$ by a suitable multiple to assume that $u \in I^{g j-g} \backslash I^{g j-g+1}$. Write $f$ for the image of $u$ in $[\mathcal{G}]_{g j-g}$. After a change of generators of $I$, which amounts to a change of variables in the polynomial ring $\mathcal{G}$, we may assume that $f \in \mathfrak{n}^{s} \mathcal{G}$ for some $s$, but that the coefficient of $x_{1}^{g j-g}$ in $f$ does not belong to $\mathfrak{n}^{s+1}$. For $\alpha \in A$ consider the $\mathcal{G}$-ideals $H_{\alpha}=$ $\left(\left\{x_{i}^{j}-\alpha x_{i}^{j-1} x_{i+1} \mid 1 \leq i \leq g-1\right\} \cup\left\{x_{g}^{j}\right\}\right)$. Since $\sqrt{H_{\alpha}}=\sqrt{\mathcal{G}_{+}}$and $H_{\alpha}$ are generated by $g$ forms of degree $j$, it follows that $H_{\alpha}$ lift to minimal reductions $J_{\alpha}$ of $I^{j}$. By [28, 2.7] one has $J_{\alpha} \cap I^{g j-g}=J_{\alpha} I^{g j-g-j}$ because $H_{\alpha}$ is generated by a regular sequence on $\mathcal{G}$. Thus if $u \in J_{\alpha}$ then $u \in J_{\alpha} I^{g j-g-j}$, which gives $f \in H_{\alpha}$. Hence it suffices to show that $f \notin H_{\alpha}$ for some $\alpha \in A$.

To do so we map $A$ onto a ring $\bar{A}$ so that $\overline{\mathfrak{n}}^{s+1}=0, \overline{\mathfrak{n}}^{s} \simeq k$, and the coefficient of $x_{1}^{g j-g}$ in $\bar{f}$ is not zero, where ' - ' denotes images in $\bar{A}$ and $\bar{A}\left[x_{1}, \ldots, x_{g}\right]$, respectively. Recall that $f \in \overline{\mathfrak{n}}^{s}\left[x_{1}, \ldots, x_{g}\right]$. Reverting to our original notation we write $A$ instead of $\bar{A}$. Now $f=a h$, with $a \neq 0$ in $A$ and $h$ a homogeneous polynomial of degree $g j-g$ that is monic in $x_{1}$.

Set $B=A\left[x_{1}, \ldots, x_{g}\right] / H_{\alpha}$ and notice that $B$ is flat, hence free, over $A$ because the minimal generators of $H_{\alpha}$ form a regular sequence on the factor ring $k\left[x_{1}, \ldots, x_{g}\right]$. Thus if $h \notin \mathfrak{n}\left[x_{1}, \ldots, x_{g}\right]+H_{\alpha}$, then the image of $h$ in $B$ is linearly independent over $A$, and hence the image of $f=a h$ in $B$ is nonzero, as desired. Finally, to show that $h \notin \mathfrak{n}\left[x_{1}, \ldots, x_{g}\right]+H_{\alpha}$ for some $\alpha \in A$, we replace $A$ by its residue field $k$. Write $M=\prod_{i=1}^{g} x_{i}^{j-1}$. For every $\alpha \in k, x_{1}^{g j-g} \equiv \alpha^{\left(\begin{array}{c}g \\ 2\end{array}\right)(j-1)} M \bmod H_{\alpha}$ and any other monomial of degree $g j-g$ in $k\left[x_{1}, \ldots, x_{g}\right]$ is either in $H_{\alpha}$ or is congruent to $\alpha^{l} M$ for some $l<\left(\begin{array}{l}g \\ 2\end{array}\right)(j-1)$. From this we conclude, first, that $M \notin H_{\alpha}$ since $[B]_{g j-g} \neq 0$. Second, $h \equiv q(\alpha) M \bmod H_{\alpha}$, where $q(Z)$ is a nonzero polynomial in $k[Z]$. Thus $q(\alpha) M \notin H_{\alpha}$ for some $\alpha \in k$, and then $h \notin H_{\alpha}$ as required.

Remark 4.3. The above proof can be simplified substantially for rings of residue characteristic zero. In conjunction with [9, 4.5], Proposition 4.2] says in particular that if $k$ is an infinite field and $R=k\left[x_{1}, \ldots, x_{d}\right]$ is a polynomial ring with irrelevant 
maximal ideal $\mathfrak{m}$, then $\left(\begin{array}{c}d j-1 \\ d-1\end{array}\right)$ general ideals generated by $d$ forms of degree $j$ intersect in $\mathfrak{m}^{d j-d+1}$.

The ideals of the next corollary have been extensively studied by S. Kleiman and B. Ulrich in 20].

Corollary 4.4. Let $R$ be a local Cohen-Macaulay ring with infinite residue field and let $I$ be a perfect $R$-ideal of height 2 . Assume that $I$ is generically a complete intersection and that $I=(\Delta, \alpha): I$ for some $\Delta \in I^{(2)}$ and some $\alpha \in I$. Then for any $j \geq 1$,

$$
\operatorname{core}\left(I^{(2 j)}\right)=I^{(4 j-1)} .
$$

Furthermore, if $R$ is Gorenstein one has $\gamma\left(I^{(2 j)}\right)=4 j-1$.

Proof. According to [20,3.3(2)], $I^{(2 j)}$ is equimultiple. Thus by [9, 3.1], $\operatorname{core}\left(I^{(2 j)}\right)$ is a finite intersection of complete intersection ideals that are reductions of $I^{(2 j)}$. Hence it suffices to check the equality core $\left(I^{(2 j)}\right)=I^{(4 j-1)}$ locally at every minimal prime $\mathfrak{p}$ of $I$. Recall that $\left(\operatorname{core}\left(I^{(2 j)}\right)\right)_{\mathfrak{p}}=\operatorname{core}\left(\left(I^{(2 j)}\right)_{\mathfrak{p}}\right)$ by $[9,4.8]$. Now $I_{\mathfrak{p}}$ is a complete intersection, and the powers and symbolic powers of $I_{\mathfrak{p}}$ coincide. The asserted equality then follows from Proposition 4.2. Finally, $\gamma\left(I^{(2 j)}\right)=4 j-1$ by Remark 2.4

\section{A Conjecture about the Core of ideAls}

We conclude the paper by analyzing a very general formula, which involves a fairly broad class of ideals. This class includes, for example, all $\mathfrak{m}$-primary (or more generally equimultiple) ideals. The thrust of Conjecture 5.1 below is to move away from the balancedness of the ideal $I$ - which is the main restriction in Theorem [2.6 - as much as possible.

Conjecture 5.1. Let $R$ be a local Cohen-Macaulay ring with infinite residue field. Let $I$ be an $R$-ideal of analytic spread $\ell \geq 1$ that satisfies $G_{\ell}$ and is weakly $(\ell-1)$ residually $S_{2}$. Let $J$ be a minimal reduction of $I$ and let $r$ denote the reduction number of $I$ with respect to $J$. Then

$$
\operatorname{core}(I)=\left(J^{r}: I^{r}\right) I=\left(J^{r}: I^{r}\right) J=J^{r+1}: I^{r} .
$$

The conjectured formula has solid theoretical foundation and an extensive computer evidence in its support. Moreover it is sharp, in the sense that [9, 4.11] provides a counterexample if the previous assumptions are relaxed. However, the situation is now more complicated than the one encountered in Theorem 2.6 as the reduction number $r$ in Conjecture 5.1 may very well depend on the chosen minimal reduction $J$ of $I$.

Example 5.2. Let $R=k[x, y]_{(x, y)}$ with $k$ a field of characteristic zero. Consider the $R$-ideals $I=\left(x^{7}, x^{6} y, x^{2} y^{5}, y^{7}\right), J=\left(x^{7}, y^{7}\right)$ and $H=\left(x^{7}, x^{6} y+y^{7}\right)$. We used the computer algebra system Macaulay and checked that $J$ and $H$ are minimal reductions of $I$ with $r_{J}(I)=4$ and $r_{H}(I)=3$, respectively. Using the algorithm we designed as a corollary of [9, 5.4] we obtained that $\operatorname{core}(I)=(x, y)^{13}$, and we also verified that $(x, y)^{13}=\left(J^{4}: I^{4}\right) I=\left(H^{3}: I^{3}\right) I$.

The next result shows that Conjecture 5.1 is also consistent with the findings of Theorem [2.6, at least in the case where the ring $R$ is Gorenstein and the powers of the ideal $I$ satisfy sliding depth conditions. 
Proposition 5.3. Let $R$ be a local Gorenstein ring with infinite residue field. Let $I$ be an $R$-ideal of height $g \geq 2$, analytic spread $\ell$, and reduction number $r$. Suppose that I satisfies $G_{\ell}$, that $\operatorname{depth} R / I^{j} \geq \operatorname{dim} R / I-j+1$ whenever $1 \leq j \leq \ell-g+1$, and that $r \leq \ell-g+1$. Then for any minimal reduction $J$ of $I$,

$$
\operatorname{core}(I)=\left(J^{r}: I^{r}\right) I=\left(J^{r}: I^{r}\right) J=J^{r+1}: I^{r} .
$$

Proof. We may assume $r \geq 1$. Choose general generators $a_{1}, \ldots, a_{\ell}$ for $J$ as in [26] 1.6(b)]. By Theorem 2.6 2 (2) we know that core $(I)=(J: I) I=(J: I) J$. According to [19, 3.4 and 4.6], the Rees algebra $\mathcal{R}(I)$ is Cohen-Macaulay and the reduction number of $I$ with respect to $J$ is $r$. But then [18, 3.4] gives the equality $J: I=$ $J I^{r-1}: I^{r}=J^{r}: I^{r}$. (Observe that $J I^{r-1}: I^{r}=J: I$ also follows from [8, 2.3]; indeed, $J I^{r-1}: I^{r}=\left(J I^{r-1}: I^{r-1}\right): I=J: I$.) Thus, we are left to show that

$$
\left(J^{r}: I^{r}\right) J=J^{r+1}: I^{r} .
$$

Clearly, $\left(J^{r}: I^{r}\right) J \subset J^{r+1}: I^{r}$. Conversely, pick $\alpha \in J^{r+1}: I^{r}$. As in the proof of Theorem 2.11 one sees that the associated graded ring $\mathcal{G}$ of $J$ is Cohen-Macaulay. Hence grade $\mathcal{G}_{+}>0$, which yields $J^{r+1}: I^{r} \subset J^{r+1}: J^{r}=J$. Thus we can write

$$
\alpha=\lambda_{1} a_{1}+\ldots+\lambda_{\ell} a_{\ell},
$$

for some $\lambda_{i} \in R$. To conclude, it suffices to show that $\lambda_{i} \in J I^{r-1}: I^{r}=J^{r}: I^{r}$ for $1 \leq i \leq \ell$. It is enough to prove the claim for $i=\ell$. Let $c$ be any element of $I^{r}$ and compare the equations

$$
\alpha c=\lambda_{1} c a_{1}+\ldots+\lambda_{\ell-1} c a_{\ell-1}+\lambda_{\ell} c a_{\ell}, \quad \alpha c=\gamma_{1} a_{1}+\ldots+\gamma_{\ell-1} a_{\ell-1}+\gamma_{\ell} a_{\ell},
$$

where $\gamma_{i} \in J^{r}$. The latter equation arises from the fact that $\alpha \in J^{r+1}: I^{r}$. In conclusion we have that

$$
\lambda_{\ell} c-\gamma_{\ell} \in\left(\left(a_{1}, \ldots, a_{\ell-1}\right): a_{\ell}\right) \cap I^{r}=\left(a_{1}, \ldots, a_{\ell-1}\right) I^{r-1},
$$

by [19, 2.5(b)]. Thus $\lambda_{\ell} c \in J I^{r-1}$ for any $c \in I^{r}$ or, equivalently, $\lambda_{\ell} \in J I^{r-1}: I^{r}=$ $J^{r}: I^{r}$ as desired.

The ideals of Proposition 4.2 provide another class of examples for which Conjecture 5.1 holds.

\section{REFERENCES}

[1] I.M. Aberbach and C. Huneke, A theorem of Briançon-Skoda type for regular local rings containing a field, Proc. Amer. Math. Soc. 124 (1996), 707-713. MR 96f:13039

[2] L. Avramov and J. Herzog, The Koszul algebra of a codimension 2 embedding, Math. Z. 175 (1980), 249-260. MR 82g:13011

[3] M. Artin and M. Nagata, Residual intersections in Cohen-Macaulay rings, J. Math. Kyoto Univ. 12 (1972), 307-323. MR 46:166

[4] D. Bayer and M.E. Stillman, Macaulay, A computer algebra system for computing in Algebraic Geometry and Commutative Algebra, 1990. Available via anonymous ftp from zariski.harvard.edu.

[5] D. Buchsbaum and D. Eisenbud, What annihilates a module?, J. Algebra 47 (1977), 231-243. MR 57:16293

[6] M. Chardin, D. Eisenbud and B. Ulrich, Hilbert functions, residual intersections, and residually $S_{2}$-ideals, Compositio Math. 125 (2001), 193-219. CMP 2001:09

[7] A. Corso and C. Polini, Reduction number of links of irreducible varieties, J. Pure Appl. Algebra 121 (1997), 29-43. MR 98h:13030

[8] A. Corso and C. Polini, On residually $S_{2}$ ideals and projective dimension one modules, Proc. Amer. Math. Soc. 129 (2001), 1309-1315. MR 2001m:13040 
[9] A. Corso, C. Polini and B. Ulrich, The structure of the core of ideals, Math. Ann. 321 (2001), 89-105. CMP 2002:02

[10] R. Cowsik and M. Nori, On the fibers of blowing-up, J. Indian Math. Soc. 40 (1976), 217-222. MR 58:28011

[11] S. Goto, S.-I. Iai and K.-I. Watanabe, Good ideals in Gorenstein local rings, Trans. Amer. Math. Soc. 353 (2001), 2309-2346. CMP 2001:08

[12] R. Hübl and C. Huneke, Fiber cones and the integral closure of ideals, Collect. Math. 52 (2001), 85-100.

[13] J. Herzog, A. Simis and W.V. Vasconcelos, Koszul homology and blowing-up rings, in Commutative Algebra, Proceedings: Trento 1981 (Greco/Valla eds.), Lecture Notes in Pure and Applied Mathematics 84, Marcel Dekker, New York, 1983, 79-169. MR 84k:13015

[14] J. Herzog, W.V. Vasconcelos and R.H. Villarreal, Ideals with sliding depth, Nagoya Math. J. 99 (1985), 159-172. MR 86k:13022

[15] J. Herzog and B. Ulrich, Self-linked curve singularities, Nagoya Math. J. 120 (1990), 129-153. MR 92c: 13010

[16] C. Huneke, Linkage and Koszul homology of ideals, Amer. J. Math. 104 (1982), 1043-1062. MR 84f:13019

[17] C. Huneke and I. Swanson, Cores of ideals in 2-dimensional regular local rings, Michigan Math. J. 42 (1995), 193-208. MR 96j:13021

[18] E. Hyry, Coefficient ideals and the Cohen-Macaulay property of Rees algebras, Proc. Amer. Math. Soc. 129 (2001), 1299-1308. MR 2001h:13005

[19] M. Johnson and B. Ulrich, Artin-Nagata properties and Cohen-Macaulay associated graded rings, Compositio Math. 103 (1996), 7-29. MR 97f:13006

[20] S. Kleiman and B. Ulrich, Gorenstein algebras, symmetric matrices, self-linked ideals, and symbolic powers, Trans. Amer. Math. Soc. 349 (1997), 4973-5000. MR 98c:13019

[21] J. Lipman, Adjoints of ideals in regular local rings, Math. Research Letters 1 (1994), 739-755. MR 95k:13028

[22] J. Lipman and B. Teissier, Pseudorational local rings and a theorem of Briançon-Skoda about integral closures of ideals, Michigan Math. J. 28 (1981), 97-112. MR 82f:14004

[23] D.G. Northcott and D. Rees, Reductions of ideals in local rings, Proc. Camb. Phil. Soc. 50 (1954), 145-158. MR 15:596a

[24] C. Polini and B. Ulrich, Linkage and reduction numbers, Math. Ann. 310 (1998), 631-651. MR 99g:13017

[25] D. Rees and J.D. Sally, General elements and joint reductions, Michigan Math. J. 35 (1988), 241-254. MR 89h:13034

[26] B. Ulrich, Artin-Nagata properties and reductions of ideals, Contemp. Math. 159 (1994), 373-400. MR 95a:13017

[27] B. Ulrich, Ideals having the expected reduction number, Amer. J. Math. 118 (1996), 17-38. MR 97b:13003

[28] P. Valabrega and G. Valla, Form rings and regular sequences, Nagoya Math. J. 72 (1978), 91-101. MR 80d:14010

[29] O. Zariski and P. Samuel, Commutative Algebra, Vol. 2, Van Nostrand, Princeton, 1960. MR 22:11006

Department of Mathematics, University of Kentucky, Lexington, Kentucky 40506

E-mail address: corso@ms.uky.edu

$U R L$ : www.ms.uky.edu/ ${ }^{\sim}$ corso

Department of Mathematics, University of Oregon, Eugene, Oregon 97403

Current address: Department of Mathematics, University of Notre Dame, Notre Dame, Indiana 46556

E-mail address: cpolini@nd.edu

$U R L$ : www.nd.edu/ ${ }^{\sim}$ polini

Department of Mathematics, Michigan State University, East Lansing, Michigan 48824

Current address: Department of Mathematics, Purdue University, West Lafayette, Indiana 47907

E-mail address: ulrich@math.purdue.edu

$U R L$ : www.math. purdue.edu/ ulrich 